\title{
Improved Albumin-Derived Per Fluorocarbon- Based Artificial Oxygen Carriers: In-vivo Evaluation of Biocompatibility
}

\author{
Anna Wrobeln, Michael Kirsch and Katja Bettina Ferenz* \\ Institute of Inorganic Chemistry, University of Duisburg-Essen, Germany
}

Submission: August 29, 2017; Published: November 27, 2017

*Corresponding author: Katja Bettina Ferenz, Institute for Physiological Chemistry, University of Duisburg- Essen, University Hospital Essen Hufelandstr, 55, 45122 Essen, Germany, Tel: +49-201-723-4106; Fax: +49 201-723-5943; Email: katja.ferenz@uk-essen.de

\begin{abstract}
Despite long lasting efforts, an artificial oxygen carrier displaying adequate gas solubility combined with a minimal risk of side effects is missing at present for clinical use, both in Europe and USA. To bypass this bottleneck, recently developed albumin-derived per fluorocarbonbased nanocapsules were further improved and tested in-vivo. Most importantly, this second nanocapsule generation reached a smaller size distribution that resulted in partially less side effects compared to the first capsule formulation. The intravenous administration to healthy rats was tolerated without any noticeable abnormalities. Systemic and blood parameters stayed within the physiological range. Minor side-effects could be observed in the release of intracellular enzymes specific for organ damage. The intravascular half -life of the second generation of albumin-derived per fluorocarbon-based nanocapsules was ameliorated with regard to the first generation. Thus, the further improved albuminderived per fluorocarbon-based nanocapsules should be tested in much more advanced and clinical relevant models
\end{abstract}

Keywords: Per fluorocarbon-based oxygen carriers; Artificial oxygen carrier; Blood substitutes; Albumin; Nanocapsules; Nano particles; Per fluorocarbon; Perfluorodecalin; Intravenous administration

Abbreviations: ALAT: Alanine Aminotransferase; ASAT: Aspartame Aminotransferase; CK: Creatine Kinase; 19F-NMR: 19F-Nuclear Magnetic Resonance Spectroscopy; HAS: Human Serum Albumin; LDH: Lactate Dehydrogenase; MAP: Mean Arterial Blood Pressure; PFC: Per Fluorocarbon; PFD: Perfluorodecalin; $\mathrm{pO}_{2}$ : Oxygen Dioxide Partial Pressure; $\mathrm{pCO}_{2}$ : Carbon Dioxide Partial Pressure; RI: Refractive Index; VIS: Viscosity

\section{Introduction}

The interest in an artificial oxygen carrier as red blood cell replacement continuously increased during the last decades [1$3]$. At the moment the necessity of blood transfusions is growing while the willingness. To donate blood is declining [4-6]. Major requirements for an artificial oxygen carrier are of course the ability to transport both, oxygen and carbon dioxide, but also the possibility to be stored easily for a long time without any loss in quality. Furthermore, the use should not be limited by any blood group characteristics and the oxygen carrier should be present in the blood circulation for a long time, i.e. some hours [7]. One way to overcome these challenges is the use of per fluorocarbons as an artificial oxygen carrier [8-11]. We developed a kind of nanocapsule to encapsulate the blood-incompatible, oxygendissolving perfluorodecalin (PFD). For that purpose, we used human serum albumin (HSA) as a membrane around the PFD core. In our previous studies, we characterized the nanocapsules, examined their functionality and performed first in-vivo biocompatibility tests $[12,13]$. The tests pointed out that the nanocapsules transport physiological relevant amounts of oxygen [12] and are able to supply hypoxic tissues [13]. The previously used nanocapsules showed slight side effects and a too short circulatory in-vivo half-life (158min) which we tried to eliminate by improving the size of the nanocapsules. In this short communication, the performance of the improved nanocapsules will be presented and compared to the first generation.

\section{Materials and Methods}

\section{Materials}

$5 \%$ human serum albumin solution (HSA 5\%, containing $5 \%$ human serum albumin, $0.75 \% \mathrm{NaCl}, 0.11 \%$ sodium-Nacetyltryptophanoate, $0.07 \%$ sodium caprylate) was purchased from Baxter

(Unterschleissheim, Germany). Perfluorodecalin (PFD) was from Fluorochem Chemicals (Derbyshire, UK). 


\section{Methods}

Synthesis of capsules: The synthesis of capsules was based on a previously described method [12] with some modifications. Here, $2.5 \mathrm{ml}$ of HSA $5 \%$ and $0.5 \mathrm{ml}$ PFD were combined in a reaction tube with a total capacity of $15 \mathrm{ml}$. The reaction tube was cooled in an ice bath and the mixture was solicited for $10 \mathrm{~min}$ using a sonotrode with a tip diameter of $3 \mathrm{~mm}$ associated with a UP 400S ultrasonic processor (Hielscher, Teltow, Germany). For sonication, the tip of the sonotrode was placed at the PFDwater interface. At a power of $400 \mathrm{~W}$, ultrasonic amplitudes with $210 \mathrm{~mm}$ and a frequency of $24 \mathrm{kHz}$ were generated.

Determination the capsule diameter: For the determination of the capsule diameter ten different batches of capsules were synthesized. Measurements were performed with a Zetasizer ZS (Malvern Instruments, Zetasizer software Version 7.11.) in intensity mode, with a $173{ }^{\circ} \mathrm{C}$ angle (noninvasive backscattering) in a clear disposable zeta cell (Malvern DTS1070) at $25{ }^{\circ} \mathrm{C}$. For the measurement, $50 \mu \mathrm{l}$ of the capsules were diluted in $950 \mu \mathrm{l}$ of the storage media. The refractive index (RI) of the capsule wall material albumin was 1.89 with absorption of 0.01 . The parameters (RI and viscosity (Vis)) of the media HSA $5 \%$ (outer phase) were as follows: RI = 1.345, Vis $1.25 \mathrm{mPa}$ s.

Biocompatibility test animals: As described in our previous study [12] 12 rats were anesthetized with isoflurane $(2.0 \%$ in $100 \%$ medical $\mathrm{O}_{2}$ at $4.0 \mathrm{l} / \mathrm{min}$ for induction, $1.5-2.0 \%$ isoflurane in $100 \%$ medical $\mathrm{O}_{2}$ at $1.0 \mathrm{lmin}$ throughout the experiment) through face masks connected to a vaporizer (Isofluran Vet Med Vapour, Draeger, Luebeck, Germany). For analgesia they received ketamine subcutaneously $(50 \mathrm{mg} / \mathrm{kg}$ body weight) into the right chest wall. After local lidocaine administration $(5 \mathrm{mg} / \mathrm{kg}$ body weight subcutaneously) a median skin-deep inguinal incision of about $2 \mathrm{~cm}$ was made along the right groin and a Portex catheter $(0.58 \mathrm{~mm}$ ID, $0.96 \mathrm{~mm}$ OD) was placed within the right femoral artery and the right femoral vein. Each catheter was fixed with a surgical suture. At the end of experiment, animals were sacrificed by resection of the heart under deep isoflurane anesthesia.

Biomonitoring: The mean arterial pressure (MAP) was recorded continuously via the femoral artery catheter. The catheter was connected to a pressure transducer and blood pressure was displayed on a monitor. Heart rates were determined from systolic blood pressure spikes and breathing rates were calculated by counting the ventilation movements per minute every $10 \mathrm{~min}$. The body core temperature of the rats was continuously monitored using a rectal sensor. A cooling below $37{ }^{\circ} \mathrm{C}$ was prevented by an underlying thermostat-controlled operating table as well as by covering the animals with wrapping film.

Assessment of blood and plasma parameters: Blood samples $(0.5 \mathrm{ml})$ for blood gas analysis as well as for the monitoring of enzyme activities in plasma were taken from the femoral artery catheter directly after catheterization of femoralis A, $3 \mathrm{~min}$ before initiation of infusion and subsequently 30,60 , $90,120,150$ and $180 \mathrm{~min}$ after start of the infusion using a $2 \mathrm{ml}$ syringe containing 80 IU electrolyte-balanced heparin (Pico50, Radiometer Medical, Brønshøj, Denmark). For each blood sampling, animals were substituted with a $0.5 \mathrm{ml}$ bolus of $0.9 \%$ $\mathrm{NaCl}$ solution via the femoral artery (with the additional effect to keep the catheter functional). In order to obtain plasma, blood was centrifuged at $4500 \mathrm{x}$ g for $10 \mathrm{~min}$ at room temperature. The gained plasma was used straight away for the determination of the activity of released enzymes.

Blood gas analysis: Arterial blood $\mathrm{pH}$, oxygen and carbon dioxide partial pressures $\left(\mathrm{pO}_{2}, \mathrm{pCO}_{2}\right)$, base excess, glucose and lactate were assessed with a blood gas analyzer (ABL 715, Radiometer, Copenhagen, Denmark).

Enzyme activities: The plasma activity of lactate dehydrogenase (LDH) as a general marker of cell injury, creatine kinase (CK) as a marker for muscle cell injury, aspartate aminotransferase (ASAT) and alanine aminotransferase (ALAT) as markers for hepatocyte injury were determined using a fully automated clinical chemistry analyzer (Vitalab Selectra E, VWR International, Darmstadt, Germany) using commercially available reagent kits (DiaSys, Holzheim, Germany).

Determination of the circulatory in-vivo half-life: After administration of improved nanocapsules $(20 \mathrm{ml} / \mathrm{kg}$ body weight $\mathrm{x}$ h) blood samples were taken from the femoral artery directly at the end of infusion and subsequently 60, 120 and $180 \mathrm{~min}$ after the end of infusion. Samples were stored at -80 ${ }^{\circ} \mathrm{C}$ until further analysis by $19 \mathrm{~F}$-nuclear magnetic resonance spectroscopy (19F-NMR) and the total amount of PFD within the samples was calculated as described previously [14]. The study group consisted of 3 animals. We assumed a virtual IV bolus model and assumed the intravascular capsule concentration at the time point $30 \mathrm{~min}=$ tmax to be $\mathrm{cmax}$.

Statistics: Data are expressed as mean values \pm SEM for invivo experiments. Comparisons among multiple groups were performed using two-way analysis of variance (ANOVA) for repeated measurements followed by Dunnett post hoc analysis. A p-value $<0.05$ was considered to indicate significance. The groups in Figure 1-3 represent $n=6$ animals. The analysis of the circulatory in-vivo half-life (Figure 4) was performed with 3 animals per group. The calculation was done by exponential onephase decay least squares ordinary fit based on the following equation:

$$
\mathrm{Y}=(\mathrm{Y} 0 \text {-plateau }) \mathrm{x} \exp (-\mathrm{K} \times \mathrm{X})+\text { plateau }
$$

The half-life was calculated with $\ln [2] / K$. For every statistical analysis, graph pad prism 6.05 (Graph Pad Software Inc, La Jolla, CA, USA) was used. 

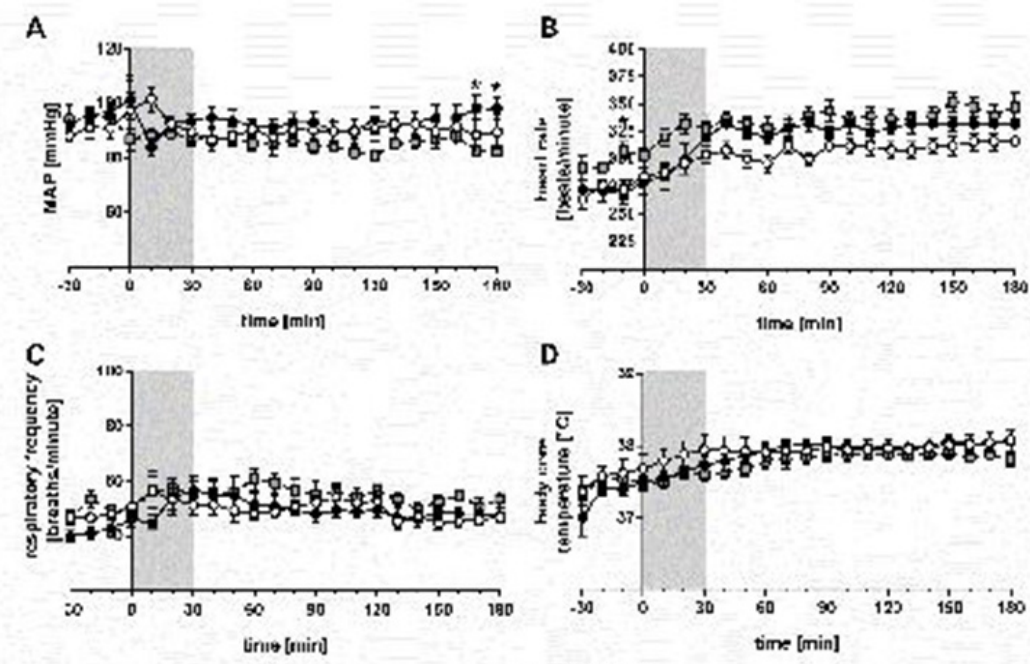

D

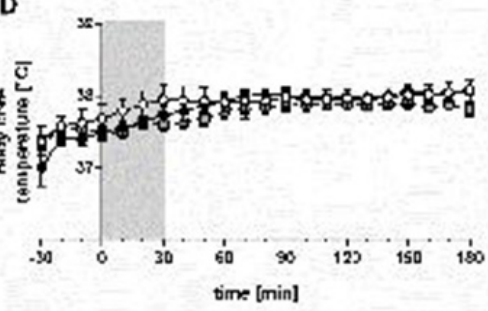

- control IHSA $5 \%$ )

- Improwed nanocapaules

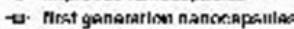

Figure 1: Effects of capsule infusion on MAP (mean arterial blood pressure) (A), heart rate (B), respiratory frequency (C) and body core temperature (D): improved nanocapsules or HSA $5 \%$ (control) were infused over a period of $30 \mathrm{~min}$ (grey, $20 \mathrm{ml} / \mathrm{kg}$ body weight $\mathrm{x} \mathrm{h}$ ). The values plotted are mean \pm SEM of 6 individual experiments per group, ${ }^{*} p<0.05$ improved nanocapsules group compared to first generation nanocapsules group.
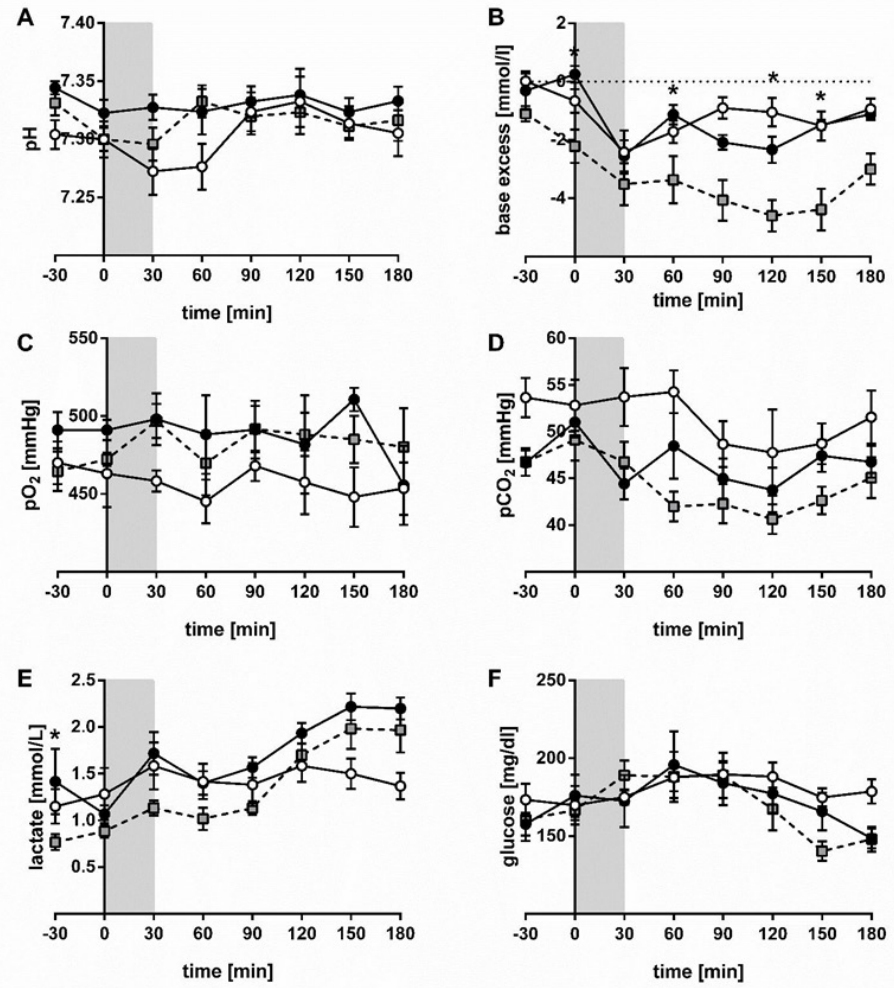

-o- control (HSA 5\%)

$\rightarrow$ improved nanocapsules

- 0 . first generation nanocapsules

Figure 2: Effects of capsule infusion on the acid base status: pH (A), base excess (B), pO2 (C), pCO2 (D), lactate (E) and glucose (F). Improved nanocapsules or HSA 5\% (control) were infused over a period of $30 \mathrm{~min}$ (grey, $20 \mathrm{ml} / \mathrm{kg}$ body weight $\mathrm{x} \mathrm{h}$ ). The values plotted are mean \pm SEM of 6 individual experiments per group, ${ }^{*} p<0.05$ improved nanocapsules group compared to first generation nanocapsules group. 

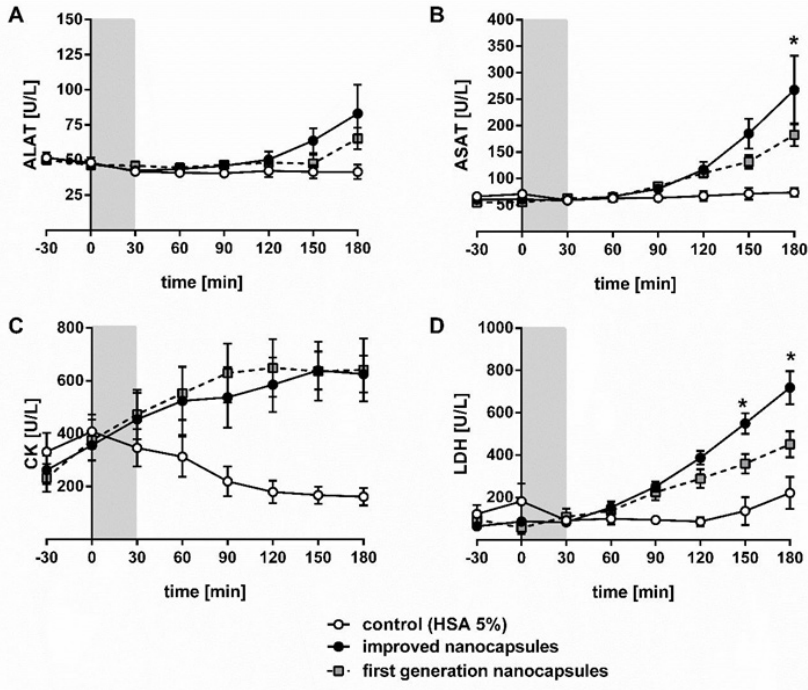

Figure 3: Effects of capsule infusion on organ/tissue damage: alanine aminotransferase (ALAT) (A), Aspartame aminotransferase (ASAT) $(B)$, creative kinase $(\mathrm{CK})(\mathrm{C})$ and lactate dehydrogenase (LDH) (D). Improved nanocapsules or HSA 5\% (control) were infused over a period of $30 \mathrm{~min}$ (grey, $20 \mathrm{ml} / \mathrm{kg}$ body weightxh). The values plotted are mean $\pm S E M$ of 6 individual experiments per group, ${ }^{*} p<0.05$ improved nanocapsules group compared to first generation nanocapsules group.

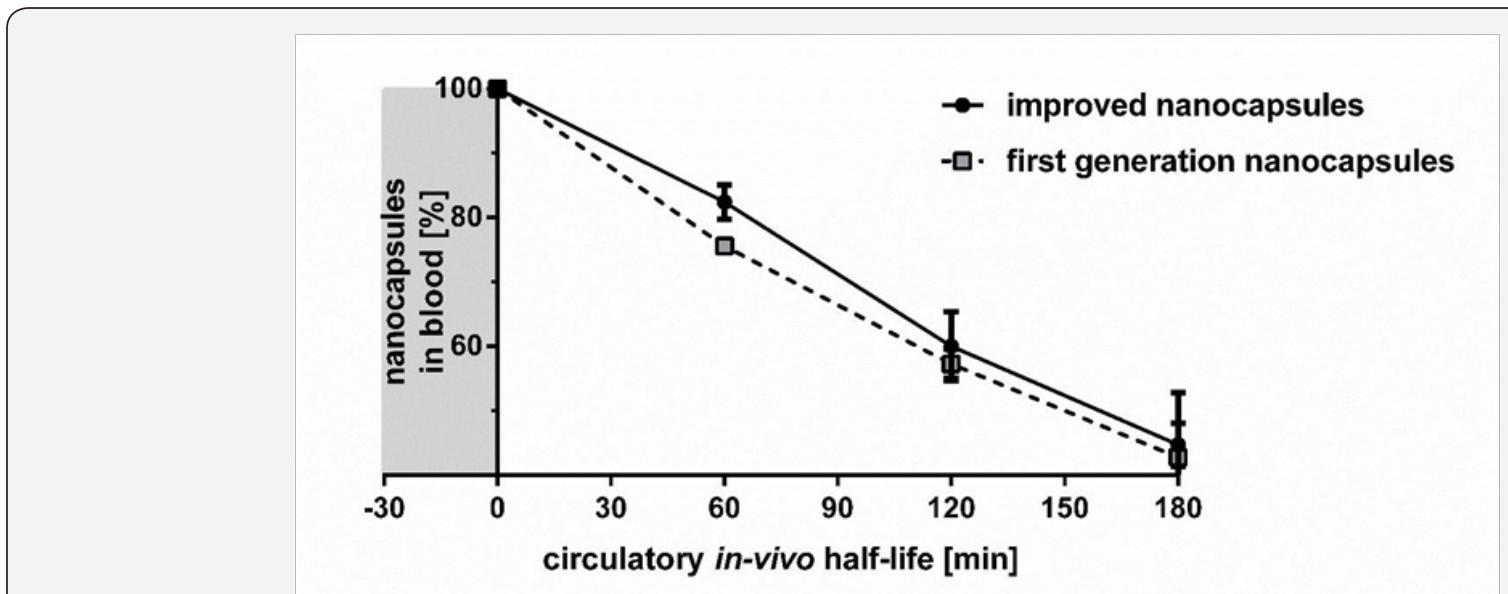

Figure 4: Circulatory in-vivo half-life: The percentage of capsules in blood was calculated over a time period of 180min post infusion. The infusion period of $30 \mathrm{~min}$ is highlighted in grey. The values plotted are mean of 3 individual experiments per group.

\section{Result}

\section{The capsule diameter}

The improvement of the synthesis created nanocapsules with a mean size of about $252 \mathrm{~nm}$. The smallest capsules showed a hydrodynamic diameter of $70 \mathrm{~nm}$ and the biggest ones $692 \mathrm{~nm}$, respectively.

\section{Biocompatibility}

The biocompatibility results of the first generation nanocapsules are reference data from our previous studies [12]. The improved nanocapsules correspond to the volumeconcentration of the first generation nanocapsules (32 Vol \%). The mapped controls were done within this work.

\section{Systemic parameters}

During the first $30 \mathrm{~min}$ of the experiment the MAP of the groups remained between $88 \mathrm{mmHg}$ and $101 \mathrm{mmHg}$ (Figure 1A). In the control group (HSA 5\%) the MAP raised immediately from $98 \mathrm{mmHg}$ to $102 \mathrm{mmHg}$ within the first $10 \mathrm{~min}$ of infusion. At the same time the animals of the improved nanocapsules group showed immediately a MAP decline from $101 \mathrm{mmHg}$ to $84 \mathrm{mmHg}$. After that the MAP remained stable between $88 \mathrm{mmHg}$ and $98 \mathrm{mmHg}$ until the end of the experiment. Before infusion, the heart rate (Figure 1B) was found between 264 and 284 beats/ minute in both groups. After the infusion animals of the improved nanocapsules group showed a partly higher heart rate of about 325 beats/minute, compared to the control animals (about 310 beats/minute). In the control group the respiratory frequency 
(Figure 1C) raised from 51 breaths/minute (immediately before the infusion) up to 57 breaths/minute within $10 \mathrm{~min}$ of infusion. The animals of the improved nanocapsules group showed a lower respiratory frequency of 45 breaths/minute under similar experimental conditions. Interestingly the respiratory frequency of the animals receiving the second generation nanocapsules (improved ones) raised up to 57 breaths/minute after an infusion period of $30 \mathrm{~min}$. After that the respiratory frequency remained stable in both groups between 51 and 54 breaths/ minute until the end of the experiment. The determined body core temperature progression (Figure 1D) was nearly identical in both groups. It rose from $37.3^{\circ} \mathrm{C}$ in the controls and $37.0^{\circ} \mathrm{C}$ in the improved nanocapsules group up to $38^{\circ} \mathrm{C}$ in both groups at the end of the experiment. Comparison between the two capsule groups revealed significant differences only in MAP (170 and180min after start of the infusion).

\section{Acid base and metabolic status}

The pH (Figure 2A) of the improved nanocapsules group was nearly unchanged over the whole experiment at a value of around $\mathrm{pH} 7.33$ whereas the animals of the control group showed a partial drop to $\mathrm{pH} 7.27$ with in the first $30 \mathrm{~min}$ of the infusion period. An increase to $\mathrm{pH} 7.32$ occurred during the next $60 \mathrm{~min}$ and from that time onwards the $\mathrm{pH}$ remained stable at this value. The control and improved nanocapsules group showed a decrease of the base excess (Figure 2B) during the period of infusion from around $0 \mathrm{mmol} / \mathrm{l}$ at the beginning of the experiment to $-2.4 \mathrm{mmol} / \mathrm{l}$ at the end of the infusion. After the infusion, the base excess fluctuated between -0.9 and $-2.3 \mathrm{mmol} / \mathrm{l}$ in both groups. After the infusion the p02 (Figure 2C) in the animals with the improved nanocapsules stayed at a value around (and in parts higher) $500 \mathrm{mmHg}$ and thus permanently above the p02 in the animals of the control group (about $450 \mathrm{mmHg}$ ). The pCO2 showed exactly the opposite behavior, because after the infusion period the $\mathrm{pCO} 2$ in the animals receiving the improved nanocapsules was lower (around $45 \mathrm{mmHg}$ ) compared to the control group with a pCO2 of about $50 \mathrm{mmHg}$. The concentration of blood lactate (Figure 2E) was constant at around $1.5 \mathrm{mmol} / \mathrm{l}$ in the control group, whereas the animals of the improved nanocapsules group showed an increase of about $2.2 \mathrm{mmol} / \mathrm{l}$ after an experimental period of $150 \mathrm{~min}$. The glucose concentration (Figure 2F) in the control group was around $180 \mathrm{mg} / \mathrm{dl}$ whereas in the animals of the improved nanocapsules group a decrease of the glucose concentration started at about $90 \mathrm{~min}$ to reach a final value of $149 \mathrm{mg} / \mathrm{dl}$. Comparison between the two capsule groups revealed significant differences only in base excess $(0,60,120$ and $150 \mathrm{~min}$ after start of the infusion).

\section{Organ/tissue damage}

Infusion of HSA $5 \%$ did not affect plasma activities of the enzymes ALAT, ASAT and CK (Figure 3A-3C). LDH showed a slight but not relevant decrease at the end of the experiment (Figure 3D) in the control group. However, plasma activities of ALAT (2 fold), ASAT (3.7 fold), CK (3.8 fold) and LDH (3.2 fold) in the improved nanocapsules group were elevated if compared to the HSA 5\% group at the end of experiment. Comparison between the two capsule groups revealed significant differences only in ASAT (180 min after start of the infusion) and LDH (150 and $180 \mathrm{~min}$ after start of the infusion), respectively.

\section{Circulatory in-vivo half-life}

The circulatory in-vivo half-life of the improved nanocapsules was calculated to be $170 \mathrm{~min}$ (Figure 4). At the end of the experiment (180min after start of the infusion) $52.9 \%$ of the improved nanocapsules were eliminated from the vascular system.

\section{Discussion}

\section{The capsule diameter}

In the present study capsules with a significantly reduced mean hydrodynamic diameter (from previously 650nm to now $252 \mathrm{~nm}$ ) and a narrower size distribution, compared to the ones used in previous studies [12], were confected in order to successfully increase the circulatory in-vivo half-life and to reduce side effects.

\section{Effects on systemic parameters}

As expected from our previous work [12] the infusion of improved nanocapsules was additionally well to legated by all animals during the experiment. In general the application did not imply dramatic changes in the recorded systemic parameters. Overall, PFC-based artificial oxygen carriers are known for transient systemic hypotension $[15,16]$. This also was confirmed for capsule-based PFC-containing systems with other wall materials than albumin $[14,17]$. Most importantly, the presented second and first generation of our nanocapsules only showed a very slight decrease of the MAP within the physiological range $(80-120 \mathrm{mmHg})$ after the infusion (Figure 1A). As expected from our study with albumin derived per fluorocarbon-based artificial oxygen carriers of the first generation, the improved nanocapsules, likewise with an albumin shell, prevented additionally systemic hypotension. The slight increase in the heart rate, compared to the control group, again within the physiological range, could be caused by the supply with oxygen (Figure 2C) after treatment with nanocapsules.

\section{Effects on acid base and metabolic status}

As expected, the improved nanocapsules operated as an artificial oxygen carrier in animals because an increase of p02 compared to the animals of the control group was clearly evident (Figure 2C). With regard to the capsules of the first generation we could not observe any difference in the p02 level [12]. In addition, the improved nanocapsules are able to remove carbon dioxide more effectively as demonstrated by the lower pCO2 compared to the control animal group (Figure 2D). Again, a nearly identical effect was observed with the nanocapsules of the first generation. 
Considering the glucose concentration, the application of first and second generation nanocapsules slightly decreased the glucose concentration (Figure 2F) [12]. Excess glycolysis could be the reason for that observation and this rationalization is supported by a corresponding slight lactate increase [18].

The animals of both the control group and the ones receiving the first generation nanocapsules, showed a clear decline in $\mathrm{pH}$ after the infusion. In contrast, the application of the second capsule generation did not lead to a decrease outside the physiological pH range (Figure 2A). That indicates a positive influence on the acid base status due to the improved nanocapsules.

The base excess in the control and improved nanocapsule group dropped to $-2.4 \mathrm{mmol} / \mathrm{l}$ but remained in the physiological range between -3 to $+3 \mathrm{mmol} / \mathrm{l}$ (Figure $2 \mathrm{~B}$ ). In our previous study the base excess fell below $-4 \mathrm{mmol} / \mathrm{l}$ (and therefore out of the physiological range) when the animals received the first generation nanocapsules [12]. Particularly regarding the base excess, the improved nanocapsules again performed better than the first generation.

\section{Effects on Organ/tissue damage}

Although the infusion of the second generation nanocapsules improved the acid base parameters, the organ tissue damage remained at similar level [12] (Figure 3).

\section{Effects on circulatory in-vivo half-life}

One major goal of developing a second nanocapsule generation was to increase the circulatory in-vivo half-life. The first generation of nanocapsules displayed a circulatory in-vivo half-life of $158 \mathrm{~min}$ [12]. In contrast, the second nanocapsules generation reached a $12 \mathrm{~min}$ longer circulatory in-vivo half-life of $170 \mathrm{~min}$ (Figure 4).

\section{Conclusion}

In this study albumin-derived per fluorocarbon- based artificial oxygen carriers were successfully improved with the result of a smaller capsule size. The second nanocapsules generation showed a good biocompatibility in a toxicological screening in an intravenous infusion model in the rat. As expected and with respect to their final destination a better oxygen supply compared to the control group could be recognized. Compared to the nanocapsules of the first generation [14] the nanocapsules of the second generation showed partially a better biocompatibility, especially in the field of acid base status was an improved result observed. Worth mentioning as well is the longer circulatory invivo half-life for the second generation nanocapsules without deterioration in biocompatibility. To sum up, the second generation albumin-derived per fluorocarbon-based artificial oxygen carriers showed slightly better biocompatibility and a longer circulatory in-vivo half-life than the first generation nanocapsules analyzed previously. The actually observed improvements are rather small but clearly indicate that the size reduction of the capsules successfully yielded in an increased circulatory in-vivo half-life and a reduction of side effects. Thus, therapeutic application of the improved nanocapsules is already conceivable even if more steps of optimization might be necessary.

\section{Acknowledgement}

We thank Prof. Matthias Epple (Institute of Inorganic Chemistry, University of Duisburg-Essen, Germany) for providing the Zetasizer ZS, Malvern Instruments

\section{References}

1. Chang TMS (2003) Future generations of red blood cell substitutes. J Intern Med 253(5): 527-535.

2. Riess JG (2001) Oxygen carriers ("blood substitutes")-raison d'etre chemistry and some physiology. Chemical Reviews 101(9): 2797-2920.

3. Weiskopf RB (2010) Hemoglobin-based oxygen carriers: compassionate use and compassionate clinical trials. Anesth Analg 110 (3): 659-662.

4. Henkel-Hanke T, Oleck M (2007) Artificial oxygen carriers: a current review. AANA J 75(3): 205-211.

5. Simoni J (2014) New approaches in commercial development of artificial oxygen. Artificial Organs 38(8): 621-624.

6. Williamson LM, Devine DV (2013) Challenges in the management of the blood supply. The Lancet 381: 1866-1875.

7. Chang TMS (2015) Red blood cell replacement or nanobiotherapeutics with enhanced red blood cell functions? Artif Cells Nanomed Biotechnol 43(3): 145-147.

8. Castro CI, Briceno JC (2010) Perfluorocarbon-Based Oxygen Carriers: Review of Products and Trials. Artifi Organs 34(8): 622-634.

9. Lowe KC (2003) Engineering blood: synthetic substitutes from fluorinated compounds. Tissue Engeenring 9(3): 389-399.

10. Sloviter HA, Kamimoto T (1967) Erythrocyte Substitute for Perfusion of Brain. Nature 216(5114): 458-460.

11. Spiess BD (2009) Perfluorocarbon emulsions as a promising technology: a review of tissue and vascular gas dynamics. J Appl Physiol 106(4): 1444-1452.

12. Wrobeln A, Laudien J, Groß-Heitfeld C, Linders J, Mayer C, et al. (2017) Albumin-derived perfluorocarbon-based artificial oxygen carriers: A physico-chemical characterization and first in vivo evaluation of biocompatibility. Eur J Pharm Biopharm 115: 52-64.

13. Wrobeln A, Schlüter KD, Linders J, Zähres M, Mayer C, et al. (2017) Functionality of albumin-derived perfluorocarbon-based artificial oxygen carriers in the Langendorff-heart. Artif Cells Nanomed Biotechnol 45(4): 723-730.

14. Laudien J, Groß-Heitfeld C, Mayer C, de Groot H, Kirsch M, et al. (2014) Perfluorodecalin-Filled Poly(n-butyl-cyanoacrylate) Nanocapsules as Potential Artificial Oxygen Carriers: Preclinical Safety and Biocompatibility. Journal of Nanoscience and Nanotechnology 15: 1-12.

15. Tremper KK, Friedman AE, Levine EM, Lapin R, Camarillo D, et al (1982) The Preoperative Treatment of Severely Anemic Patients with a Perfluorochemical Oxygen-Transport Fluid, Fluosol-DA. The New England Journal of Medicine 307(5): 277-283.

16. Waxman K, Cheung CK, Mason GR (1984) Hypotensive reaction after infusion of a perfluorochemical emulsion. Crit Care Med 12(7): 609610. 
17. Ferenz KB, Waack IN, Laudien J, Mayer C, Broecker-Preuss M, et al (2014) Safety of Poly (ethylene glycol)-coated Perfluorodecalin-filled Poly (lactide-co-glycolide) microcapsules following intravenous administration of high amounts in rats. Results in Pharma Sciences 4: 8-18.
18. Phypers B, Pierce JMT (2006) Lactate physiology in health and disease. Continuing Education in Anaesthesia Critical Care \& Pain 6(3): 128132.

\section{Your next submission with Juniper Publishers will reach you the below assets}

- Quality Editorial service

- Swift Peer Review

- Reprints availability

- E-prints Service

- Manuscript Podcast for convenient understanding

- Global attainment for your research

- Manuscript accessibility in different formats

( Pdf, E-pub, Full Text, Audio)

- Unceasing customer service

Track the below URL for one-step submission https://juniperpublishers.com/online-submission.ph 